\title{
A rare presentation of subarachnoid hemorrhage: Vertical gaze palsy - A case report
}

\author{
Gülşah CI*, Seda D, Hikmet SC and Yunsur C \\ Department of Emergency Medicine,University of Health Sciences Keçiören Training and Research Hospital, Ankara, Turkey
}

\begin{abstract}
This case of vertical gaze palsy due to mesencephalon hemorrhage represents an unusual presentation of subarachnoid hemorrhage (SAH).

A 28-year-old male patient presented with acute onset of headache and vertical diplopia. On admission he had no neurological deficit except for slightly limited vertical eye movements of the left eye. Three hours after onset, he was slightly lethargic; vertical gaze palsy became more prominent; ptosis developed, which was more evident on the left side; and the left pupil became mydriatic. He became unconscious and was taken for an operation for ventricular drainage, but he died. Magnetic resonance imaging (MRI) angiography of the brain showed tortuous vascular structures in the posterior mesencephalon, evaluated as arteriovenous malformation.
\end{abstract}

Conclusion: Spontaneous midbrain hemorrhages are very rare but extremely life-threatening clinical conditions that most frequently present with oculomotor disorders such as ptosis and vertical gaze palsy. In young SAH patients, MRI angiography is considered the gold standard to elucidate the underlying etiology, which is vascular malformation most of the time.

\section{Introduction}

Subarachnoid hemorrhages (SAHs) are uncommon but lifethreatening causes of headache with a median case fatality rate of 27-44\% [1]. Isolated spontaneous midbrain hemorrhages are very rare clinical entities and by far most frequently present together with oculomotor disorders [2]. The oculomotor nerve has general somatic efferent and general visceral efferent components, and the main features of oculomotor palsy are ptosis, diplopia, limited eye movements, and pupillary dysfunction [3]. The leading causes of midbrain hemorrhages are vascular malformations, and about one-third of the patients with posterior communicating artery (PCoA) aneurysms present with oculomotor nerve palsy $[2,3]$.

Here, we describe the case of a patient presenting with vertical gaze palsy secondary to mesencephalon hemorrhage.

\section{Case presentation}

A 28-year-old male patient presented to the emergency department with acute onset of headache and vertical diplopia. On admission, the patient was alert (Glasgow Coma Scale (GCS) score of 15) and had no neurological deficit except for slightly limited vertical eye movements of the left eye. His vital signs were within normal limits: blood pressure, 140/75 mmHg; pulse rate, 82 beats/min; temperature, $36.8{ }^{\circ} \mathrm{C} ; \mathrm{SpO}_{2}$, $96 \%$; and blood glucose, $75 \mathrm{mg} / \mathrm{dL}$. There were no vascular risk factors or comorbid diseases in the patient's medical history. Computed tomography revealed a hyperdense nodular lesion of $4 \mathrm{~mm}$ in diameter in the left mesencephalon, which could have been a hemorrhage or a mass lesion. In diffusion-weighted magnetic resonance imaging (MRI), T2 axial images showed diffuse and enlarged vascular structures in the posterior mesencephalon and edema in the left mesencephalon (Figure 1). The patient consulted with neurology and neurosurgery. Antiemetic and mannitol were delivered via a peripheral intravenous cannula for symptomatic relief and to decrease the intracerebral edema.
Upon examination 3 hours after onset, he was slightly lethargic but able to follow simple verbal commands. Vertical gaze palsy became more prominent, ptosis developed and was particularly evident on the left side, the left pupil became mydriatic, and the pupils were bilaterally unresponsive to light. The patient then started to vomit and became unconscious. His GCS score dropped to 8 and he was intubated. To clarify the etiology, MRI and MRI angiography were planned. MRI showed a contrasted lesion in the posterior mesencephalon, which was possibly an arteriovenous malformation, and diffuse hemorrhage in the 3rd and 4th ventricles and also in the lateral ventricles and sulcus. There was hydrocephalus due to intraventricular hemorrhage. MRI angiography of the brain was unremarkable except for tortuous vascular structures in the posterior mesencephalon (possibly an arteriovenous malformation) and hemorrhage in the ventricular system (Figure 2). The patient was taken for an operation for ventriculostomy drainage but suffered arrest during the operation and died.

\section{Discussion}

The leading causes of midbrain hemorrhages that usually present with third nerve palsy are vascular malformations (40\%), hypertension (25\%), and bleeding diathesis (5\%) [2]. However, the diagnosis of subarachnoid and intracerebral hemorrhages in young adults is challenging, and according to the study of Mathon et al., common vascular malformations other than sacciform aneurysm and

*Correspondence to: Gulsah Cikrikci Isik, Department of Emergency Medicine,University of Health Sciences Keçiören Training and Research Hospital, Ankara, Turkey, Tel: +90 543765 6176; E-mail: gulsah8676@gmail.com

Key words: vertical gaze palsy, ptosis, midbrain hemorrhage, subarachnoid hemorrhage, arteriovenous malformation

Received: August 20, 2020; Accepted: September 02, 2020; Published: September 09, 2020 

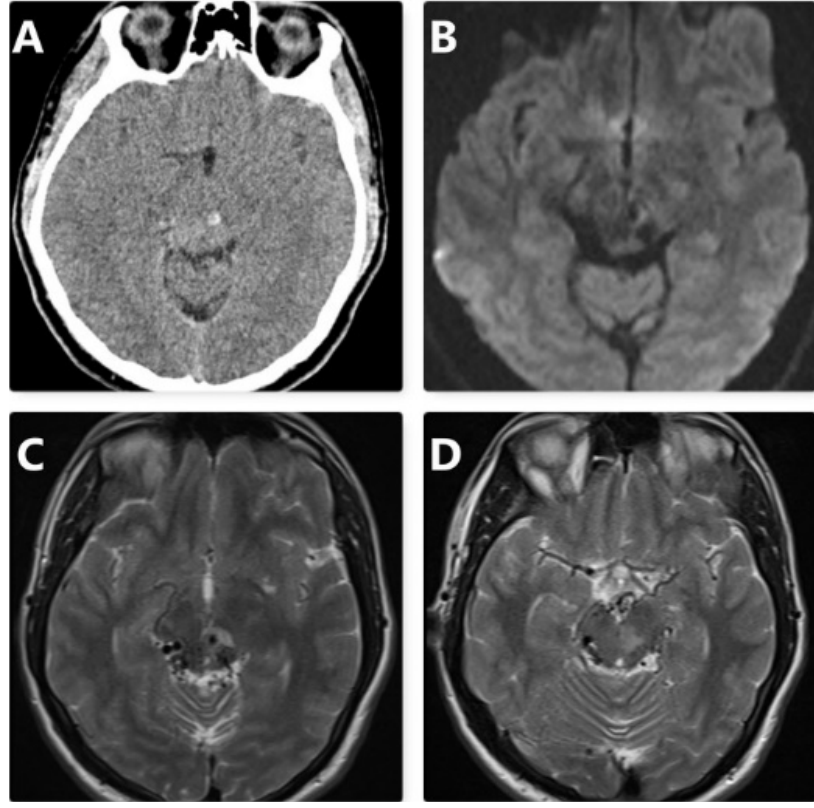

Figure 1. Computed tomography revealed a hyperdense nodular lesion $4 \mathrm{~mm}$ in diameter in the left mesencephalon (hemorrhage? mass lesion?) (A); Diffusion-weighted image (B); T2 axial images $(\mathrm{C}, \mathrm{D})$ showed diffuse and enlarged vascular structures in the posterior mesencephalon and edema in the left mesencephalon
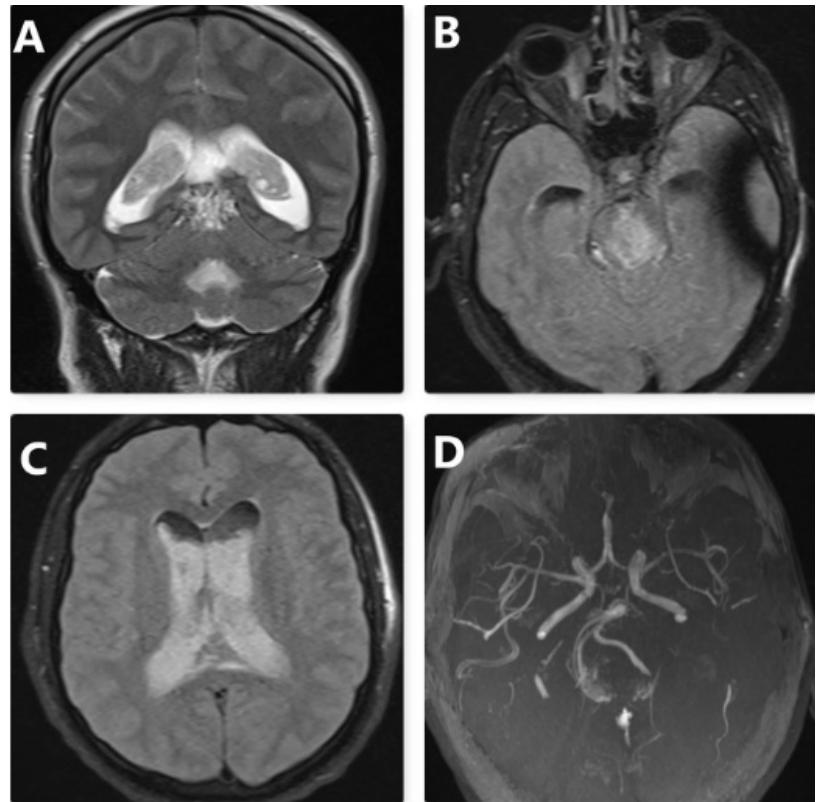

Figure 2. T2 coronal images showed a contrasted lesion in the posterior mesencephalon (arteriovenous malformation?) (A); T2 fluid-attenuated inversion recovery (FLAIR) images showed the diffuse hemorrhage in the ventricles $(\mathrm{B}, \mathrm{C})$; MRI angiography of the brain was unremarkable except for tortuous vascular structures in the posterior mesencephalon (arteriovenous malformation?) (D)

arteriovenous malformations were reversible cerebral vasoconstriction syndrome, cerebral venous sinus thrombosis, and mycotic aneurysms [4]. MRI and formal digital subtraction angiography (DSA) are the gold standards in young SAH patients to elucidate the underlying etiology in order to offer optimal management care [4]. Our patient was a young male who also presented with findings of third nerve paralysis such as vertical gaze palsy and ptosis; he was diagnosed with mesencephalon hemorrhage due to arteriovenous malformation with MRI angiography.
Oculomotor nerve paralysis (ONP), which commonly presents with diplopia and ptosis, has various and complex etiological factors such as subarachnoid hemorrhage, tumors, cavernous sinus lesions, pituitary lesions, painful ophthalmoplegia, and even diabetes mellitus [3]. Intracranial aneurysm is the leading vascular cause of ONP associated with SAH and ruptured aneurysms were more frequently internal carotid artery and basilar artery aneurysms [5].

Understanding the anatomy and physiology of the eye and the connection of the cranial nerves related to vision and eye movements is very important to understand neuro-ophthalmologic emergencies [6]. The central caudal nucleus, which is located in the midbrain, contains bilateral levator neurons and has a role in vertical eye movements, so damage to this region may result in eyelid ptosis and vertical gaze palsy [7]. However, the topographical arrangement of the oculomotor fibers is very complicated and the supranuclear pathways involved in the vertical gaze are not well understood yet. Case reports in the literature have presented vertical gaze palsy with the diagnosis of unilateral thalamic infarction or putaminal hemorrhage $[8,9]$. Therefore, vertical gaze palsy, which was previously believed to be a midbrain lesion, may be attributed to a broader spectrum of anatomical areas now.

\section{Conclusion}

Spontaneous midbrain hemorrhages are very rare but extremely life-threatening clinical conditions that most frequently present with oculomotor disorders such as ptosis and vertical gaze palsy. In young SAH patients, MRI angiography is the gold standard to elucidate the underlying etiology, which is vascular malformation most of the time. Therefore, emergency physicians must know the basics of neuroophthalmologic system examination and the interpretation of findings in order to offer optimal management care.

\section{References}

1. Marcolini E, Hine J (2019) Approach to the diagnosis and management of subarachnoid hemorrhage. West J Emerg Med 20: 203-211. [Crossref]

2. Moncayo J (2012) Midbrain infarcts and hemorrhages. Front Neurol Neurosci 30:158 161. [Crossref]

3. Raza HK, Chen H, Chansysouphanthong T, Cui G (2018) The aetiologies of the unilateral oculomotor nerve palsy: a review of the literature. Somatosens Mot Res 35: 229-239. [Crossref]

4. Mathon B, Ducros A, Bresson D, Herbrecht A, Mirone G, et al. (2014) Subarachnoid and intra-cerebral hemorrhage in young adults: rare and underdiagnosed. Rev Neurol 170:110-118. [Crossref]

5. Aiba T, Fukuda M (2003) Unilateral oculomotor nerve paresis associated with anterior communicating artery aneurysm rupture--two case reports. Neurol Med Chir 43: 484487. [Crossref]

6. Huff JS, Austin EW (2016) Neuro-ophthalmology in emergency medicine. Emerg Med Clin North Am 34: 967-986. [Crossref]

7. Togay Işıkay C, Polat BS (2016) Oculomotor nerve palsy presented with isolated unilateral ptosis and minimal upgaze palsy. Neuroophthalmology 40: 86-89. [Crossref]

8. Khan M, Sidiropoulos C, Mitsias P (2011) Unilateral thalamic infarction presenting as vertical gaze palsy: A case report. J Med Case Rep 5: 535. [Crossref]

9. Kanda T, Azuma K, Sakai F (2002) Vertical gaze palsy associated with bilateral ptosis following right putaminal hemorrhage. J Stroke Cerebrovasc Dis 11:113-116. [Crossref]

Copyright: (C)2020 Gülşah CI. This is an open-access article distributed under the terms of the Creative Commons Attribution License, which permits unrestricted use, distribution, and reproduction in any medium, provided the original author and source are credited. 